\title{
Fault Tolerant Coverage Model for Sensor Networks
}

\author{
Doina Bein ${ }^{1}$, Wolfgang W. Bein ${ }^{2}$, and Srilaxmi Malladi ${ }^{3}$ \\ 1 School of Computer Science, University of Nevada Las Vegas, NV \\ siona@cs.unlv.edu \\ 2 School of Computer Science, University of Nevada Las Vegas, NV \\ bein@cs.unlv.edu \\ 3 Department of Computer Science, Georgia State University, GA \\ cscsrmx@cs.gsu.edu
}

\begin{abstract}
We study the coverage problem from the fault tolerance point of view for sensor networks. Fault tolerance is a critical issue for sensors deployed in places where are not easily replaceable, repairable and rechargeable. The failure of one node should not incapacitate the entire network.
\end{abstract}

We propose three 1 fault tolerant models, and we compare them among themselves, and with the minimal coverage model [8].

Keywords: Coverage, fault tolerance, smart sensors, sensor network.

\section{Introduction}

If all sensors deployed within a small area are active simultaneously, an excessive amount of energy is used, redundant data is generated, and packet collision can occur on transmitting data. At the same time, if areas are not covered, events can occur without being observed.

A density control function is required to ensure that a subset of nodes is active in such a way that coverage and connectivity are maintained. Coverage refers to the total area currently monitored by active sensors; this needs to include the area required to be covered by the sensor networks. Connectivity refers to the connectivity of the sensor network modeled as a graph: the currently active sensors has to form a fully connected graph such that the collected data can be relayed to the initiators (the nodes requesting data).

We study the coverage problem from the fault tolerance point of view. Fault tolerance is a critical issue depending on where the sensors are employed. Sensors coupled with integrated circuits, known as smart sensors, provide high sensing from their relationship with each other and with higher level processing layers. A smart sensor is specificaly designed for the targeted application [4. Smart Sensors find their applications in a wide variety of fields such as military, civilian, bio-medical as well as control systems, etc. In military applications, sensors can track troop movements and help decide deployment of troops. In civilian 
applications, sensors can typically be applied to detect pollution, burglary, fire hazards and the like.

It is clear that fault tolerance is improtant when maintaining survivability in such applications. The failure of one node should not incapacitate the entire network. Wireless body sensors implanted in the body must be energy efficient, utilize bandwidth, robust, lightweight and fault tolerant as they are not easily replaceable, repairable and rechargeable. Bio sensors need a dynamic, self-stabilizing network.

Motivation and Contributions. We are interested in the bio-medical domain where applications of sensors are relatively new. Sensors are already applied to monitor temperature level, glucose level, organs and its implants, and to detect external agents in the body in connection with cancer and other health abnormalities. We note that for such body chips to work properly, two-way communication between external computer controlling sensors and the actual sensors is needed. There may be tens and hundreds of sensors gathering data, thus wireless are preferable over wired sensors.

The goal of our paper is to propose several sensor array placement schemes, which are fault tolerant. Thus despite the presence of limted number of failed sensors, the system continues to function. We propose three 1 fault tolerant models, and we compare with each other and with the minimal coverage model 8 .

Outline of the Paper. In Section 2, we review related work which has motivated our paper. Section 3 presents the various parameters for the sensor nodes and describes their relationship to our models, which are further described in Section 4. In Secton 5 we compare those models, and we finish with concluding remarks in Section 5.

\section{Related Work}

The nodes in a wireless environment are greatly dependent on the battery life and power. Therefore, minimizing energy consumption for the network while keeping its functionality is a major objective in designing a robust, reliable network. But sensors are prone to failures and disconnection. Only minimal coverage of a given region without redundancy would make such a network inattractive from a practical point of view. Therefore it is necessary to not only design for minimal coverage, on the other hand fault tolerance features must viewed in light of the additional sensors and energy used.

Given a sensor network deployed in a target area, [2] focused on finding whether each point of the area is covered by at least $K$ sensors. [9] extends the problem further and focuses on selecting a minimum size set of sensor nodes which are connected in such a way that each point inside the area covered by the entire sensor network is covered by at least $K$ sensors.

Starting from the uniform sensing range model [8], two models are proposed using sensors with different sensing ranges [7. Variable sensing range is novel, 
unfortunately both models are worse in terms of achieving a better coverage. Also, the second model in [7] requires (for some sensors) that the communication range to be almost six times larger than the sensing range, otherwise connectivity is not achieved.

A relay node, also called in the literature, gateway [1] or application node [5], acts as clusterhead in the corresponding cluster. In [3] a fault-tolerant relay node placement scheme is proposed for wireless sensor networks, and a polynomial time approximation algorithm is presented to select a set of active nodes, given the set of all the nodes.

In 6] the project of building a theoretical artificial retina made up of smart sensors is described. The sensors should form a tapered array that should rests on retina and produce electrical signals which are converted by the underlying tissue into chemical signals to be sent to the brain. The sensor array is therefore used for both reception and transmission in a feedback system. The challenges with these sensors are the wireless networking, distribution, placement and continuing operation of these sensors.

\section{Preliminaries}

Two parameters are important for a sensor node: the wireless communication range of a sensor $r_{C}$, and the sensing range $r_{S}$. They generally differ as values, and a common assumption is that $r_{C} \geq r_{S}$. Obviously, two nodes $u$ and $v$, whose wireless communication ranges are $r_{C_{u}}$, respectively $r_{C_{v}}$, can communicate directly if $\operatorname{dist}(u, v) \leq \min \left(r_{C_{u}}, r_{C_{v}}\right)$.

In [8], it is proven that if all the active sensor nodes have the same parameters (radio range $r_{C}$ and sensing range $r_{S}$ ) and the radio range is at least twice of the sensing range $r_{C} \geq 2 \times r_{S}$, complete coverage of an area implies connectivity among the nodes. Therefore, under this assumption, the connectivity problem reduces to the coverage problem.

There is a trade-off between mimimal coverage and fault tolerance. For the same set of sensors, a fault tolerant model will have a smaller area to cover. Or, given an area to be covered, more sensors will be required, or the same number of sensors but with a higher values for the parameters.

A model is $k$ fault tolerant if by removal of any $k$ nodes, the network preserves its functionality. A $k$ fault tolerant model for the coverage problem will be able to withstand $k$ removals: by removing any $k$ nodes, the covered region remains the same. A 0 tolerant model will not work in case of any removal of a node.

A straightforward approach is to either double the number of sensors in each point, or to double the sensor parameters for some sensors of the minimal coverage model to make it 1 tolerant. Similar actions can be taken for a 1 tolerant model to be 2 tolerant and so on. In order for a $k$ fault-tolerant model to be worthwhile, it has to be better than the straightforward approach.

We propose three 1 fault tolerant models, and we compare them among each other, and with the minimal coverage model in 8 . 


\section{Fault Tolerant Models}

For all models, we assume the sensing range to be $r$, and we compare them among each other, and with the minimal coverage model [8]. In the first model, the basic structure is composed of four sensors arranged in a square-like structure of side $r$. In the second model, the basic structure is composed of six sensors arranged in a regular hexagon-like structure of side $r$. In the third model, the basic structure is composed of seven sensors arranged in a regular hexagon-like structure of side $r$, and the center of the hexagon as well. In these models the assumption that the communication range is greater than twice the sensing range guarantees the connectivity of the network.

\subsection{Square Fault Tolerant Model}

The basic structure for the first model is drawn in Figure 1 $(a)$.

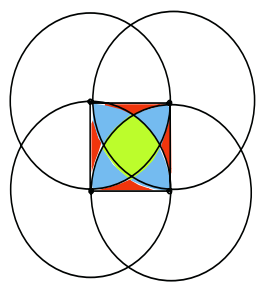

(a) Four sensors in a square arrangement

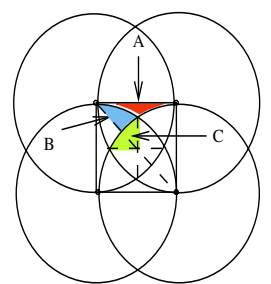

(b) Selected areas $A, B$, and $C$

Fig. 1. Square fault tolerant model

The square surface $S_{4}=r^{2}$ is partitioned into an area covered by exactly two sensors $S_{2 s}^{\text {square }}$, an area covered by exactly three sensors $S_{3 s}^{\text {square }}$, and an area covered by exactly four sensors $S_{4 s}^{\text {square }}$.

In order to calculate the values for those areas, let $A, B$, and $C$ to be some disjoint areas as drawn in Figure $1(b)$. We observe that $S_{2 s}^{\text {square }}=4 S_{A}, S_{3 s}^{\text {square }}=$ $8 S_{B}, S_{4 s}^{\text {square }}=4 S_{C}$.

We can derive the following system of equations:

$$
\begin{aligned}
\left\{\begin{array} { l } 
{ S _ { A } + 2 S _ { B } + S _ { C } = \frac { r ^ { 2 } } { 4 } } \\
{ S _ { B } + S _ { C } + \frac { 1 } { 4 } = \frac { \Pi r ^ { 2 } } { 8 } } \\
{ 4 S _ { B } + 4 S _ { C } + S _ { A } = \frac { \Pi r ^ { 2 } } { 3 } - \frac { r ^ { 2 } \sqrt { 3 } } { 4 } }
\end{array} \Rightarrow \left\{\begin{array}{l}
S_{A}=r^{2}-\frac{r^{2} \sqrt{3}}{4}-\frac{\Pi r^{2}}{6} \\
S_{B}=-\frac{r^{2}}{2}+\frac{r^{2} \sqrt{3}}{4}+\frac{\Pi r^{2}}{24} \\
S_{C}=\frac{r^{2}}{4}-\frac{r^{2} \sqrt{3}}{4}+\frac{\Pi r^{2}}{12}
\end{array}\right.\right. \\
\Rightarrow\left\{\begin{array}{l}
S_{2 s}^{\text {square }}=4 r^{2}-r^{2} \sqrt{3}-\frac{2 \Pi r^{2}}{3} \\
S_{3 s}^{\text {square }}=-4 r^{2}+2 r^{2} \sqrt{3}+\frac{\Pi r^{2}}{3} \\
S_{4 s}^{\text {square }}=r^{2}-r^{2} \sqrt{3}+\frac{\Pi r^{2}}{3}
\end{array}\right.
\end{aligned}
$$


Therefore, given a 2D-region of dimension $(r N) \times(r M)$, with $N$ and $M$ strictly positive integers, we can derive the following results. The number of sensors required is $(N+1) \times(M+1)$. The ratio between the sensor area used and the area covered is $\frac{(N+1)(M+1) \Pi r^{2}}{N M r^{2}}=\frac{(N+1)(M+1) \Pi}{N M}$. The area covered by two sensors is $N M S_{2 s}^{\text {square }}=N M\left(4 r^{2}-r^{2} \sqrt{3}-\frac{2 \Pi r^{2}}{3}\right)$.

The area covered by three sensors is $N M S_{3 s}^{\text {square }}=N M\left(-4 r^{2}+2 r^{2} \sqrt{3}+\frac{\Pi r^{2}}{3}\right)$.

The area covered by four sensors is $N M S_{4 s}^{s q u a r e}=N M\left(r^{2}-r^{2} \sqrt{3}+\frac{\Pi r^{2}}{3}\right)$.

\subsection{Hexagon Fault Tolerant Model}

The basic structure for the second model is drawn in Figure $2(a)$.

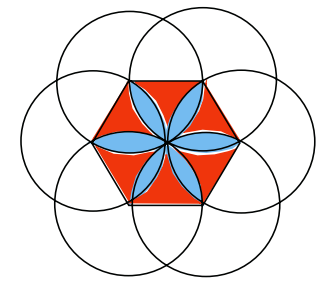

(a) Six sensors in a regular hexagon arrangement

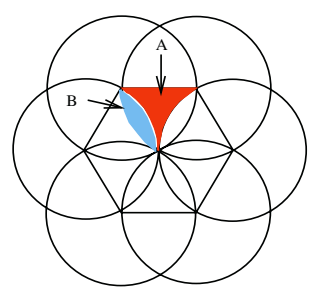

(b) Selected areas $A$ and $B$

Fig. 2. Hexagon fault tolerant model

The hexagon surface $S_{6}=\frac{3 \sqrt{3}}{2}$ is partitioned into an area covered by exactly two sensors $S_{2 s}^{\text {hexagon }}$, and an area covered by exactly three sensors $S_{3 s}^{\text {hexagon }}$.

In order to calculate the values for those areas, let $A$ and $B$ be some disjoint areas as drawn in Figure $2(b)$. We observe that $S_{2 s}^{\text {hexagon }}=6 S_{A}$ and $S_{3 s}^{\text {hexagon }}=$ $6 S_{B}$.

We can derive the following system of equations:

$$
\begin{gathered}
\left\{\begin{array} { l } 
{ S _ { A } + S _ { B } = \frac { r ^ { 2 } \sqrt { 3 } } { 4 } } \\
{ \frac { 1 } { 2 } S _ { B } + \frac { r ^ { 2 } \sqrt { 3 } } { 4 } = \frac { \Pi r ^ { 2 } } { 6 } }
\end{array} \Rightarrow \left\{\begin{array}{l}
S_{A}=\frac{3 r^{2} \sqrt{3}}{4}-\frac{\Pi r^{2}}{3} \\
S_{B}=\frac{\Pi r^{2}}{3}-\frac{r^{2} \sqrt{3}}{2}
\end{array}\right.\right. \\
\Rightarrow\left\{\begin{array}{l}
S_{2 s}^{\text {hexagon }}=\frac{9 r^{2} \sqrt{3}}{2}-2 \Pi r^{2} \\
S_{3 s}^{\text {hexagon }}=-3 r^{2} \sqrt{3}+2 \Pi r^{2}
\end{array}\right.
\end{gathered}
$$

Therefore, given a $2 \mathrm{D}$ region of dimension $(r N) \times(r M)$, with $N$ and $M$ strictly positive integers, we can derive the following results. The number of sensors required is $N\left\lceil\frac{2 M}{s q r t 3}\right\rceil$. 
The ratio between the sensor area used and the area covered is $\frac{N\left\lceil\frac{2 M}{s q r t 3}\right\rceil \Pi r^{2}}{N M r^{2}}=$ $\frac{\Pi\left\lceil\frac{2 M}{s q r+3}\right\rceil}{M}$.

The area covered by two sensors is $\frac{1}{6} N\left\lceil\frac{2 M}{\text { sqrt3 } 3} S_{2 s}^{\text {hexagon }}=\frac{1}{6} N\left\lceil\frac{2 M}{\text { sqrt3 }}\right\rceil\left(\frac{9 r^{2} \sqrt{3}}{2}-\right.\right.$ $\left.2 \Pi r^{2}\right)$. The area covered by three sensors is $\frac{1}{6} N\left\lceil\frac{2 M}{\text { sqrt3 }}\right\rceil S_{3 s}^{\text {hexagon }}=$

$$
\frac{1}{6} N\left\lceil\frac{2 M}{s q r t 3}\right\rceil\left(-\overline{3} r^{2} \sqrt{ } 3+2 \Pi r^{2}\right) .
$$

\subsection{Improved 7-Node Model}

We now consider instead of the minimal coverage model of three nodes, the seven-node model obtained by overlapping three three-node models (see Figure 3) $(a)$, and we call it as the improved model.

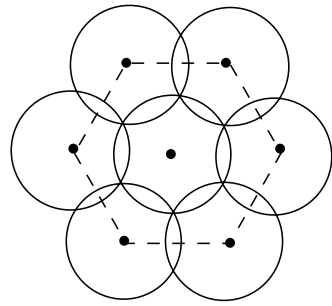

(a) 7-node minimal coverage model

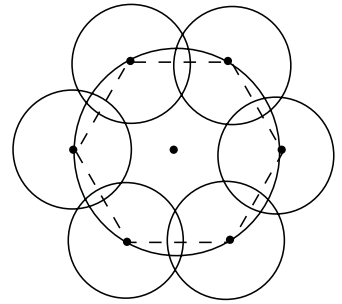

(b) Fault tolerant improved 7-node model

Fig. 3. 7-node models

The minimal coverage model can be made 1 fault tolerant by modifying the sensing range of some nodes: one of the three sensors increases its sensing range from $r$ to $r \sqrt{3}$. In this manner, when we overlap three such models we obtain the improved 7 -node model (see Figure $3(b)$ ).

Therefore, given a $2 \mathrm{D}$ region of dimension $(r N) \times(r M)$, with $N$ and $M$ strictly positive integers, we can derive the following results. The number of sensors required is $\left(\left\lfloor\frac{N}{\sqrt{3}}\right\rfloor+1\right) \times\left(\left\lfloor\frac{2 M}{3}\right\rfloor+1\right)$.

\section{Comparative Results}

We consider the following models: the minimum coverage model [8], the improved model, the square model, and the hexagonal model. Let $f$. $t$. be a short form of fault tolerant.

We compare the models in terms of number of active nodes at a time required for covering a certain area, the covered area given a fixed number of nodes, and probability to function. Consider that all the sensors, independent of their sensing range, have the probability $p$ to fail, $0 \leq p \leq 1$, therefore the probability 
to function is $1-p$. Also we assume that any two failures are independent one another.

In Table 1, we consider the following notations. Covered area denotes the area covered by the polygonal line formed by the sensors. Fraction used denotes the fraction of the sensor areas used for covering that area; this value aids in calculating the energy used for covering the region. Efficiency is defined as the ratio between the previous two values (the covered area and the fraction of the sensor area used), and denotes the efficiency of using a particular model. Max. nodes to fail denotes the maximum number of nodes that can fail and still the coverage is available over the number of nodes in the model. Prob. to function denotes the probability for the model to be functional.

The values for the probability functions in case of the square and hexagonal model from Table 1 follow. The probability to function in case of square model is $P_{\text {square }}=(1-p)^{4}+4 p(1-p)^{3}+2 p^{2}(1-p)^{2}=(1-p)^{2}\left(1+2 p-p^{2}\right)$. In case of the hexagonal model, the probability to function is $P_{\text {hexa }}=(1-p)^{6}+6 p(1-$ $p)^{5}+15 p^{2}(1-p)^{4}+2 p^{3}(1-p)^{3}=(1-p)^{2}\left(1+3 p+6 p^{2}-8 p^{3}\right)$.

Table 1. Comparisons among the four models

\begin{tabular}{|ccccc|}
\hline & $\begin{array}{c}\text { Min. cov. } \\
0 \text { f. t. }\end{array}$ & $\begin{array}{c}\text { Improved } \\
1 \mathrm{f} \text {. t. }\end{array}$ & $\begin{array}{c}\text { Square } \\
1 \mathrm{f} . \mathrm{t} .\end{array}$ & $\begin{array}{c}\text { Hexagonal } \\
1 \mathrm{f} \text {. t. }\end{array}$ \\
\hline No. sensors & 7 & 7 & 4 & 6 \\
model covered area & $\frac{9 \sqrt{3} r^{2}}{2}$ & $\frac{9 \sqrt{3} r^{2}}{2}$ & $r^{2}$ & $\frac{3 r^{2} \sqrt{3}}{2}$ \\
fraction used & $3 \Pi r^{2}$ & $5 \Pi r^{2}$ & $\Pi \Pi r^{2}$ & $2 \Pi r^{2}$ \\
efficiency & $\frac{3 \sqrt{3}}{2 \Pi} \simeq 0.827$ & $\frac{9 \sqrt{3}}{10 \Pi} \simeq 0.496$ & $\frac{1}{\Pi} \simeq 0.318$ & $\frac{3 \sqrt{3}}{4 \Pi} \simeq 0.413$ \\
max. nodes to fail & $0 / 6$ & $6 / 7$ & $2 / 4$ & $3 / 6$ \\
prob. to function & $(1-p)^{7}$ & $1-p+p(1-p)^{6}$ & $P_{\text {square }}$ & $P_{\text {hexa }}$ \\
\end{tabular}

From Table 1 we observe that the minimal coverage model has the best efficiency, followed by the improved, hexagonal, and square model. Also, we observe that the hexagonal model has the highest probability to function, followed by the square, improved, and the minimal model.

\section{Conclusion}

We study the coverage problem from the fault tolerance point of view for sensor networks. Fault tolerance is a critical issue for sensors depending on where the 
sensors are employed. The failure of one node should not incapacitate the entire network. Wireless body sensors have to be energy efficient, utilize bandwidth, robust, lightweight and fault tolerant as they are not easily replaceable, repairable and rechargeable.

We propose three 1 fault tolerant models, and we compare them among themselves, and with the minimal coverage model. We are currently working on algorithms to move sensors in order to preserve the network functionality when more than a fault occurs. If the network layout is composed by hundreds of such proposed models, in some cases sensors need to be moved to cover areas left uncovered by faulty or moving sensors.

\section{References}

1. G. Gupta and M. Younis. Fault-Tolerant clustering of wireless sensor networks. In Proceedings of IEEE Wireless Communications and Networking Conf. (WCNC), pages $1579-1584$.

2. C.F. Huang and Y.C. Tseng. The coverage problem in a wireless sensor network. In ACM Intl. Workshop on Wireless Sensor Networks and Applications (WSNA), pages 115-121, 2003.

3. B. Hao, J. Tang, and G. Xue. Fault-tolerant relay node placement in wireless sensor networks: formulation and approximation. In IEEE Workshop on High Performance Switching and Routing (HPSR), pages 246-250, 2004.

4. A. Moini. Vision chips or seeing silicon. In Department of Electrical and Electronics Engineering, University of Adelaide, Australia, http://www.iee.et.tu-dresden.de/iee/eb/analog/ papers/mirror/visionchips/vision_chips/smart_sensors.html, 1997.

5. J. Pan, Y.T. Hou, L. Cai, Y. Shi and S.X. Shen. Topology control for wireless sensor networks. In Proceedings of ACM MOBICOM, pages 286-299, 2003.

6. L. Schwiebert, S.K.S. Gupta, and J. Weinmann. Research challenges in wireless networks of biomedical sensors. In ACM Sigmobile Conference, pages 151-165, 2001.

7. J. Wu and S. Yang. Coverage issue in sensor networks with ajustable ranges. In Intl. Conf. on Parallel Processing (ICPP), pages 61-68, 2004.

8. H. Zhang and J.C. Hou. Maintaining sensing coverage and connectivity in large sensor networks. In Proceedings of NSF Intl. Workshop on Theoretical and Algorithmic Aspects of Sensor, Ad Hoc Wireless, and Peer-to-Peer Networks, 2004.

9. Z. Zhou, S. Das, and H. Gupta. Connected k-coverage problem in sensor networks. In Intl. Conf. on Computer Communications and Networks (ICCCN), pages 373378, 2004. 